\title{
Ableitung des periodischen Systems der chemischen Elemente aus der Elektronentheorie.
}

\author{
Von Heingich Tetdot. \\ Mit 14 Figuren im 'Text.
}

\section{Die grundlegenden Anschanungen über den Aufbau der Atome and Yoleküle ans Elektronen.}

bekanntlich haben Lntersuchungen von Ruthraroro und Bome zu der Vorstellung geführt, $\mathrm{da} B$ ein Atom aus einem elektrisch positiv geladenen Atomkern besteht, dor von negativen Elektronen umkreist wird. Da diese Elektronen sich gegenseitig absto Ben, müssen sie sich so weit entfernt voncinander einstellen, wie es die Anziehungskraft des Atomkerns zuläßt. Da ferner der Atomkern auf sämtliche Elektronen eine gleich gro $\beta_{A}$ Anziehungskraft ausübt, müssen diese bestrebt sein, sich auf einer Kugelfläche einzustellen, in deren Mittelpunkt dieser Atomkern liegt. Dabei müssen die Elektronen mit solcher Goschwindigkeit kreisen, dab ihre Zentrifugalkraft der Anziehungskraft des Atomkerns die Wage hält, weil sie sonst in das Innere des Atomkerns gezogen würdon. Um diese Zentrifugalgeschwindigkeit zu erreichen, suchen die Elektronen sich möglichst nahe am Äquator der genannten Kugelfläche einzustellen, so dab die beiden Pole dieser Kugelfläche frei von Elektronen bleiben. Diese beiden Pole werden daher infolge der von positiven Atomkern ausgehenden Kraftlinien elektrisch positiv.

Schon vor den eben erwähnten Arbeiten von RuTHrarord und Born wurde von J. Stan ${ }^{1}$ ) eine Hypothese aufgestellt, nach welcher die Verbindung zweior Atome zu einem Molekül mit Hilfe von Valenzelektronen erfolgt. Bei einwertigen Verbindungen löst sich :sus jedem Atom ein Valenzelektron, bei zweiwertigen Verbindungen zwei Valenzelektronen usw. Diese Valenzelektronen stellen sich zwischen die Atome und halten dieselben dadurch zasammen, dab rin Teil ihrer Kraftlinien sich an positive Sphären des einen Atoms and ein anderer Teil ihrer Kraftlinien sich an positive Sphären des anderen Atoms anheftet. Diese positiven Sphären müssen nach den

J) Jahrbuch der Radioaktivität und Elektronik 1908. Bd. V. S. 124-163. 
vorhergehenden Ausführungen an den beiden Polen der den Atomkern umgebenden Elektronenkugelfläche liegen.

Die von den Stakzechen Valenzelektronen ausgehenden Kraftlivipn müssen daher nach einem der beiden Pole der das Atom begrenzenden Kngelfläche gehen und werden durch diesen Pol hindurch von dem im Zentrum des Atoms befindlichen positiven Kern angezogen, vgl. Fig. 1.

Forner muB man annehmen, daB sich die den Atomkern umgebenden Elektronen symmetriech auf der eben besprochenen Kugelnäche nm dem Atomkern zueinander tinstellen und auch während

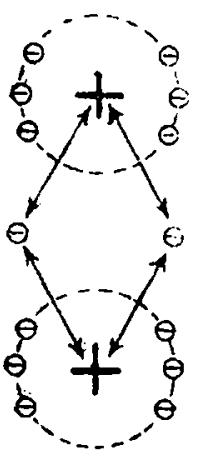

Fig. 1. ihrer Umdrehung in symmetrischer Stellung zueinander bleiben. Denn sonst könnte ein derartiges sus sich bewegenden Elektronen bestehendes Gebilde unmöglich so beständig sein, wie es ein Atom ist. Aus dieser Forderung der Symmetrie folgt, $\mathrm{d} a \mathrm{~B}$ die zu cinem Atom gehörenden Elektronen in zueinander parallelen Bahnen und mit gleicher Winkelgeschwindigkeit $v m$ ihren Atomkern kreisen. Verbinden sich nun zwei gleichartige Atome des. selben Elementes mitcinander, so werden sie sich so zucinander einzustellen suchen, dafo sowohl die Kreisbahnen der um ihre beiden Atomkerne krejsenden Elektronen als auch die Kreisbahn der zwischen ihnen kreisenden Valenzelektronen parallel zueinander sind. Zwei durch Valenzelektronen Verbindung zweier Atome $\mathrm{zo}$ einem Molekuil darch Valenzelektronen. su einem Molekül miteinander verbundene Atome müssen sich daher in der aus Fig. 1 ersiehtlichen Weise gurinander einstellen.

\section{Die Fratstohung der Perioden im periodisohen System der Blomonte.}

Wir wollen nun die Atome der verschiedenen Elemento betrachten. Dabei na $B$ folgendes vorausgeschickt worden: da jedes Atom nach anßen hin neutral erscheint, müssen die negativen Elektronen und positiven Ladungen des Atomkerns sich gegenseitig aufheben; daraus folgt, daB die Zahl der. am Atomkern vorhandenen positiven Ladungen ebenso groß sein muB wie die Zahl der zu diesem Atom gehörenden Elektronen. Diese Zahl entepricht nun nach Versachen, die Rutrenrond mit Wrbonschen Aufnahmen und MosswY mit Röntgenstrahlen angestellt hat, der Stellung, die ein Bleunent in dem ron Mandelsmpr nnd L. Mryer entdeckten perio- 
disehen System der Elemente einnimmt; d.h. also, das erste Element, der Wasserstoft, hat 1 Elektron, das zweite Element, das Helinm, hat 2 Elektronen, das dritte Element, das Li. hat 3 Elektronen usw. (vgl. Tabelle auf Seite 192).

Da zum Wasserstoffatom nur ein einziges Flektron gehört, bildet (in Wasserstoffatom, wie Fig. 2 zeigt, kein symmetrisches Gebilde und ist daher für sich allein nicht genügend existenzfähig. Dagegen entsteht ein symmetrisches und existenzfähiges Gebilde, wenn zwei Wasserstoffatome sich zu einem Molekül verbinden, indem ihre beiden Elektronen als Valenzelektronen zwischen die Atome treten, so daB das aus Fig. 3 ersichtliche Gebilde entsteht.

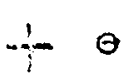

Fig. 2.

Wasererstoffatom.

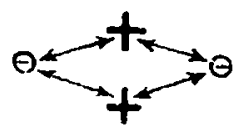

Fig. 3.

Wasserstoffmolekül.

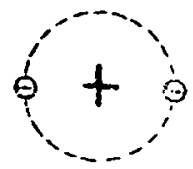

Fig. 4.

Inneres Elektronensystem des Helinms und der übrigen Elemente der ersten Horizontalreilie.

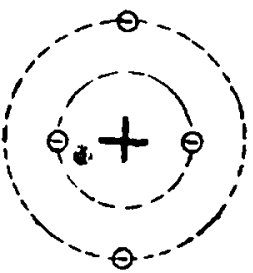

Fig. 5. Inneres and außeres Elektronensystem eines Koblenstoffatoms bei $\mathrm{zw}$ eivertigen Kohlenstoffverbindungen.

Bei dem auf den Wasserstoff folgenden Element, dem Helium, wird der Atomkern von 2 Elektronen umkreist, die sich, wie Fig. 1 zeigt, symmetrisch zum Atomkern einstellen können. Infolge dieser Symuetrie ist das Heliumatom im Gegensatz zam Wasserstoffatom beständig. Wenn aber beim Heliumatom eines der beiden Elektronen vom Atomkern fort in eire zwischen zwei Atomkernen betindliche Valenzstellung gehen würde, so würde das Heliumatom dadurch ebenso unsymmetrisch und wenig existenzfähig werden, wie das für sich allein nicht existenzfähige Wasserstoffatom. Aus diesem Grunde kann das Helinmatom kein Valenzelektron abgeben, mit dessen Hilfe es sich mit einem enderen Atom za einem Molekül verbinder könnte.

Auf das Helium folgen (vgl. die Tabelle auf S. 192) das Li mit 3 Elektronen, das Be mit 4 Elektronen und das B mit 5 Elektronen. Da das Li einwertig ist, geht 1 Elektron von seinen 3 Elektronen in die Valenzstellung, und es bleiben am Atomkern 2 Eloktronen zurück; beim Be, das zweiwertig ist, gehen 2 Elektronen in 


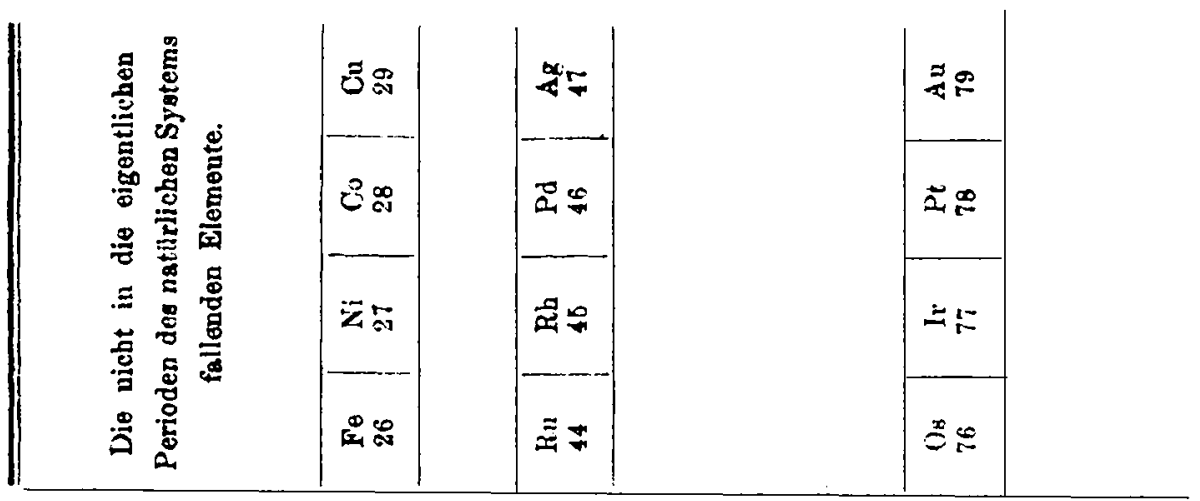

\begin{tabular}{|c|c|c|c|c|c|c|c|c|c|c|c|}
\hline 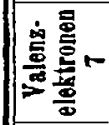 & 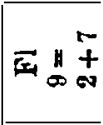 & ठ & 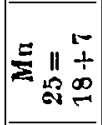 & 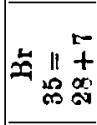 & 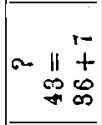 & 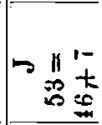 & & & & 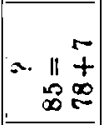 & \\
\hline 离总 & 0 & $\infty \begin{array}{l}n+ \\
0 \\
0\end{array}$ & $5 \stackrel{+}{11+}+$ & 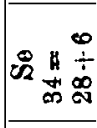 & 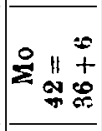 & 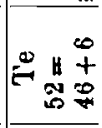 & & & & $=\begin{array}{l}11+0 \\
+\infty \\
\infty\end{array}$ & $=\underset{\infty}{\infty} \underset{\infty}{\infty}+\infty$ \\
\hline 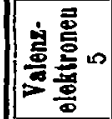 & $\pi \stackrel{n+\infty}{n+\infty}$ & 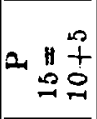 & 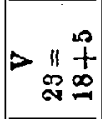 & 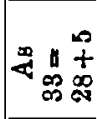 & $\frac{0}{2} \frac{11}{7}$ & 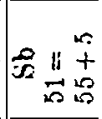 & & & & 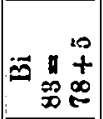 & $\frac{1}{6+\infty}$ \\
\hline 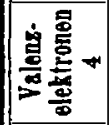 & 0 it & $\ddot{\infty}=$ & $=\begin{array}{ll}11 & + \\
-\alpha & +\end{array}$ & 象早芒 & $\dot{\mathbf{N}} \underset{\sim}{11}+$ & $=\frac{1}{1}+\frac{+}{0}$ & & & & 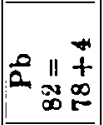 & $\begin{array}{l}n+ \\
-1 \\
8 \infty \\
8 \infty\end{array}$ \\
\hline 兽总 & $\prod_{1}^{11+}$ & 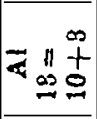 & 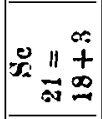 & की & 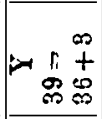 & $=\begin{array}{l}u^{\circ}+ \\
0 \\
0\end{array}$ & & & & $\vec{\infty} \frac{n}{\infty}$ & $\int_{0} n^{\infty}+$ \\
\hline 兽总总 & 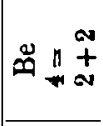 & $\underbrace{\infty}_{0} \mu \stackrel{+}{n+}$ & (n) & 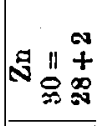 & 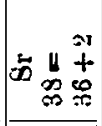 & 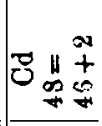 & 至 & & & 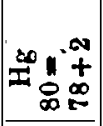 & 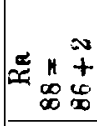 \\
\hline 总总 & $\because \underset{+}{\|}$ & $\Rightarrow+$ & $\underset{11}{11}+$ & & 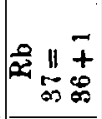 & & $\begin{array}{lll}3 & 11 & \overrightarrow{+} \\
0 & 5 & 5\end{array}$ & & & & $\therefore \underset{\infty}{n+\infty}$ \\
\hline 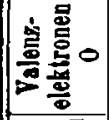 & 类 & 总 & $\begin{array}{r}0 \\
4 \underset{1}{0}+ \\
\infty \\
0\end{array}$ & & 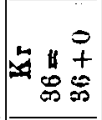 & & 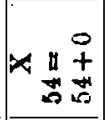 & & & & $\begin{array}{cc}5 & 0 \\
-1 & + \\
0 & 0 \\
\infty & 0\end{array}$ \\
\hline 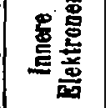 & N & $\therefore$ & $\stackrel{\infty}{=}$ & $\stackrel{\infty}{\infty}$ & $\stackrel{8}{*}$ & 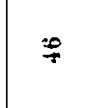 & $\vec{z}$ & & & $\stackrel{\infty}{\infty}$ & $\mathscr{0}$ \\
\hline & $\rightarrow$ & or & (1) & $*$ & $\therefore$ & 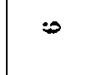 & r. & $\infty$ & $\infty$ & $\cong$ & $\vec{E}$ \\
\hline
\end{tabular}


die Valenzstellung, und es bleiben ebenfalls 2 Elektronen am Atom. bern zurück; beim B., das dreiwertig ist, gehen 3 von den vorhandenen 5 Elektronen in die Vulenzstellung und es bleiben wiederum 2 Elektronen am Atomkern zurück. Bei jedem der eben genannten, in der ersten Horizontalreihe stehenden Elemente sind also 2 Elektronen so fest mit den Atomkern verbunden, daB sie ebenso wie die beiden Elektronen des Heliums nicht von diesem fort in eine Valenzstellung gehen künuen.

Der nun folgende Kohlenstoff hat 6 Elektronen, von denen bei vierwertigen Kohlenstoffverbindungen 4 vom Atomkern fort in eine Valenzstellung gehen, so daB auch hier ebenso wie bei den vorhergehenden Elementen dieser Horizontalreihe noch 2 Elektronen am Atomkern bleiben, die niemals von diesem fortgehen. Dagegen bleiben bei den zweiwertigen Kohlenstoffverbindungen noch 4 Elektronen arn Atomkern zurück. Diese werden aber mit verschieden grober Kraft vom Atomkern festgehalten. Denn zwei von ihnen können, sobald das Kohlenstoffatom eine vierwertige Verbindung eingelt, vom Atomkern fort in eine Valenzstellung gehen, während die beiden anderen Elektronen so fest mit dem Atomkern verbunden sind, $d a B$ sie ebensowenig wie die beiden Elektronen des Heliums yon ihrem Atomkern fort in eine Valenzstellung gehen können. Diese verschieden starke Bindung läBt sich dadurch erklären, daB dio beiden fester am Atomkern haftenden Elektronen in einer inneren Kreis-oder Ellipsenbahn, die beiden weniger fest haltenden Elektronen in einer äuBeren Kreis- oder Ellipsenbahn um ihren Atomkern kreisen (Fig. 5).

Auch bei den drei noch übrig bleibenden Elementen der ersten Horizontalreihe, dem $\mathrm{N}, \mathrm{O}$ und $\mathrm{Fl}$, sind jedesmal zwei Elektronen so fest mit ihrem Atomkern verbunden, $\mathrm{daB}$ sie nicht von diesem weg in eine Valenzstellung gehen können. Säıntliche Elemente der ersten Horizontalreihe besitzen also ein inneres Elektronensystem von zwei Elektronen, die so fest mit dem Atomkern verbunden sind, $\mathrm{da} B$ sie uuf keinen Fall von dinsem fort in eine Valenzstellung gehen können. Dagegren können die übrigen zum Atom gehörenden Elektronen sämtlich vom Atomkern fort in eine Valenzstellung gehen. Sie tun dies b:i den gesättigten Sauerstoff verbindungen. Bei den übrigen Verbindungen geht aber nur ein Teil dieser Valenzelektronen vom Atomkern fort in die Valenzstellung und die übrigen Valenzelektronen bleiben beim Atomkern und kreisen wie beim Kohlenstoff (Fig. 5) in einen äuBeren Ring um den Atomkern. Für diesen äuBeren Ellek- 
tronenring bleiben bei den dreiwertigen Stickstoffverbindungen 2 Elektronen übrig, bei den zweiwertigen Sauerstoffverbindungen 4 and bei den einwertigen Fl-Verbindangen (Fig. 6) 6 Flektronen.

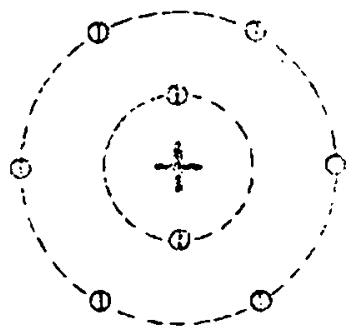

Fig. 6.

Inneres und äaberes Elektronensystem eines Fluorstoms bei einwertigen Fluorverbindangen.

Wenn man die folgenden Elemente in dieser Wcise weitcr betrachtet, so stellt sich heraus, daß sämtliche Elemente der zweiten Horizontalreihe ein inneres Elektronensystem von 10 Elektronen haben, die unter kcinen Unständen vom Atomkern fort in eine: Valenzstellung gehen können. Die Elemente der dritten Horizontadreihe haben ein inneres Elektronensystem von 18 Elektronen, die Elemente der vierten Horizontalreihe haben ein inneres Elektronensystem von 28 Elektronen usw., vgl. die Tabelle auf 8: 192.

Die aoht Perioden des natürlichen Systems der Elemente kommen demnach dadurch zustande, daß sämtliche Elemente einer Horizontalreihe eine glcichgro Be Anzahl innere Elektronen und sämtliche Elemente einer Vertikalreihe eine gleichgroße Anzahl äußerer Elektronen haben. Die Zahl der inneren Elektronen ist in der Tabelle auf S. 192 am Anfang jeder Horizontalreihe, die Zahl der äußeren Elektronen am Kopf jeder Vertikalreihe angegeben.

Die Elemente, die in ciner Vertikalreihe untereinander stehen, zeigen also deshalb $t$ in glcichartiges chemisches Verhalten, weil sie eine gleichgroBe Anzahl änBerer Elektronen oder Valenzelektronen haben, welche die Wertigke it der Verbindungen, die diese Elemente eingehen, bestimmen.

In diescr Arbait soll nun noob gezeigt werden, wie die Entstehung eines metallischen oder metalloiden Charakters sowie das Atomvolumen in erster Linie bestimnt wird durch die Stellung, die die Elektronen dfs inneren Elektronensystems zum Atomkerm und aueinander einnehmen. Bei den rechts stehenden Elementen kommt dann zu dem inneren Elektronensystem noch ein äuBerer 
Elloktronenring hinzo, dessen Entstehung im Vorhergehenden, bei den rechtsstehenden Elementen der ersten Horirontale (C, N, O, FI) abgeleitet wurde, der aber in gleicher Weise ouch bei den rechtsstehenden Elementen der folgenden Horizontalreihen entsteht. Auch die Elektronen dieses äuBeren Elektronenringes bei den reohtsstehenden Elementen üben cinen Einflu $B$ auf die Bildung des metallischen oder metalloiden Charakters sowie auf das Atomvolumen ans. In welcher Weise diese Wirkungen des inneren Elektronensystems und des bei den rechts stehenden Elementen hinzukommenden änBeren Elektronenringes zustande bommen, soll in den folgenden Kapiteln abgeleitet werden.

\section{Entstehung des metallischen and metalloiden Charakters der Elemento.}

Zunächst soll angenommen werden, daB Metalle und Metalloide sich dadurch voneinander unterscheiden, da $B$ aus einem Metallatom Elektronen leichter herausgesto Ben werden können, als aus einem Metalloidatom. Diese Annahme ist nicht neu, denn man hat sehon die metallische Lritung des elektrischen. Stromes dadurch erklärt, da $B$ Elektronen von Atom zu Atom wandern und dabci ihre elektrischen Ladungen weiter tragen. Die wandernden Elektronen können aber nicht durch die Atome hindurchkommen, sondern stoßen gegen dieselben und stoßen dabei aus ihnen andere Elektronen heraus, deren Stelle sie dann einnehmen, während die herausgesto Benen Elektronen die Weiterführung des elektrischen Stromes bewirken. Diese Art der Wciterleitung des /elektrischen Stromes ist bei den Metallen deshalb möglich, weil bei ihnen die Elektronen nur relativ schwach von ihrem Atomkern angezogen werden, so daB sie durch die hinzukommenden neuen Elektronen herausgestoßen werden können. Dagegen können die Mictalloide den elektrischen Strom nicht weiterleiten, weil in ihren Atomen sämtliche Elektronen so stark vom Atomkern angezogen werden, $\mathrm{da} B$ sie nicht aus dem Atom herausgestoßen werden können.

Es sollen zunächst diejenigen Horizontalreihen des periodischen Systems betrachtet werden, in denen der metallische Charakter der Elemente von links nach rechts regelmäBig abnimmt; $\epsilon$ sind dies die erste, zweite, vierte and sechste Horizontalreibe. Die Abnahmo des metallischen Charakters in diesen $R \in$ ihen ist nach den Ausführungen des vorhergehenden Absatzes darauf zurückzuführen, daB die Kraft, mit welcher die Elektronen vom Atomkern angegogen 
werden, bei den Elementen difser Rcihen von links nach reahts zunimmt, so dab die Elektronen immer schwerer herausgestoßen werden können. Diese Znnahme der von den Atomkernen ausgeübten Ansiehnngskräfte läBt sich aus der hier entwickelten Elektronentheorie in folgender Weise ableiten:

Da die inneren Elektronen dem Atomkern näher sind als die änBeren, müssen auch die positiven Ladungen des Atomkerns auf die inneren Elektronen stärker $c$ inwirken als arf die änBeren. Die Zahl der inneren Elektronen ist aber nur bei dem ersten Element jeder Horizontalreihe ebenso groß wie die Zahl der positiven Ladungen am Atomkern. Dann wächst die Zahl der positiven Ladungen von Element zu Element, während die Zahl der inneren Elektronen bei den Elementen jeder Horizontalreihe konstant bleibt. So werden z. B. die beiden inneren Elektronen der ersten Horizontalreihe beim He von 2 positiven Ladungen am Atomkern festgehalten, beim Ii von 3 positiven Ladungen, beim $B$ von 4 , und so steigt die $Z$ ishl der positiven Ladungen weiter bis die beiden inneren Elektronen beim Fl von 9 positiven Larlungen des Atomkerns festgehalten werden. In der zweiten Horizontalreihe werden die 10 Elektronen des inneren Elektronensystems beim $\mathrm{Na}$ von 11 positiven Ladungen beim $\mathrm{Cl}$ von 17 positiven Ludungen fistgehalten usw.

Jo mehr die Zahl der positiven Ladungen eines Atomkerns über die Zahl der diesen umkreisenden inneren Elektronen hinauswächst, desto größer muß die Zentrifugalkraft dieser inneren Elektronen werden, damit dicse nicht in den Atomkern hineingezogen werden. Kommt nun zu den inneren Elektronen noch ein äuBerer Elektronenring hinzu, so müssen dessen Elektronen sich mit derselben Winkelgeschwindigkeit wie die inneren Elektronen drehen, da sonst Störungen in dem System sich bewegender Elektronen, die das Atom bilden, eintreten würden. Die Umdrehungsgesch windigkeit sämtlicher einen Atomkern umkreisenden Elektronen muB daher innerhalb jeder Horizontalreihe von links nach rechts zunehmen, nnd je größer diese Unidrehungsgeschwindigkeit wird, desto schwerer lassen sich die Elektronen aus der Kreisbahn, in der sie um ihren Atomkern kreisen, herausstoBen. Je schwerer sie sich aber aus ihrem Atomkern herauss to Ben lassen, desto schwächer wird nach dem vorher Gesagten der metallische Charakter der Elemente. Es müBte daher cigentlioh in allen Horizontalrihen eine Abnahme des metallischen Charakters der Elemente von links nach rechts stattfinden. Tatsächlich aber tritt diese Abnahme nu in der ersten, zweiten, vierten 
upd sechsten Reihe deutlich zum Ausdruck. Bei den übrigen Horizontalreihen müssen daher noch andere Umstände binzukommen, die bewirken, daB Elektronen aus den Atomen der dort rechtsstehenden Elemente herausgestoßen werden können, so dab diese Elemento einen metallischen charakter erhalten.

Die eine dieser Ursachen besteht darin, dab die Wahrschein. lichkeit, daB einzelne Elektronen aus dem Atom herausgestoBen werden können, um so mehr wächst, je gröBer die Zabl der zu einem Atom gehörenden Elektrorien wird. Aus diesem Grunde haben von der siebenten Horizontalreihe $a b$ fast alle Elemente einen metallischen Oharabter.

$A u$ Berdem mu $B$ noch eine andere Ursache vorhanden sein, welche bewirkt, daß auch die rechtsstehenden Elemente der dritten und fünften Horizontalreihe einen metrllischen Charakter haben, während die ihnen entsprechenden Elemente der ersten, zweiten, vierten und sechsten Horizontalreihe Metalloide sind. Diese Ursache ist darauf zurückznführen, daß die Elektronen des äuBeren Elektronenringes, dessen Entstehung im vorhergehenden Kapitel abgeleitet wurde, bei den Metalloiden der ersten; zweiten, vierten und sechsten Horizontalreihe vom Atombern so fest gehalten werden, $d_{a} B$ sie nicht aus dem Atomverbande herausgestoßen wexden können, während sie bei den entsprechenden Elementen der fünften und dritten Hojizontalreihe weniger fest mit dem Atomkern verbunden sind. Wie dies kommt, soll in folgendem gezeigt werden.

Bei den Elementen der ersten Horizontalreibe müssen die Kraft. linien auf ihrem Wege vom Atomkern zu den Elektronen des äuBeren Ringes zwar durch den inneren Elektronenring hindurchgehen, werden dabei aber nicht oder doch nur unbedeutend gestört, da im inneren Ringe nur zwei Flektronen vorhanden sind, die sich infolge der gegenseitigen AbstoBung der Elektronen möglichst weit von den äuBeren Elektronen entfernt einstellen (vgl. Fig. 5 und 6), so daB sie stets außerhalb des graden Verbindungsweges zwischen deut Atomkern und einem der äußeren Elektronen liegen.

Bei dem rechtsstehenden Elementen der ersten Forizontalreihe stehen daher nicht nur die Elektronen des inneren Elektronensystems, sondern auch dio Elektronen des äuberen Elektronenringes mit dem Atomkern in ungestörter direkter Verbindung und werden daber so stark von diesem angezogen, $\mathrm{da} B$ sie nicht vom Atomkern fort gestoben werden können. Da bei diesen rechtsstehenden Elementen der ersten Horizontalreihe demnach keine fortstobbaren 2. soorg. a allg. Chem. Bd. 106. 
Elektronen vorhanden sind, können diese Elemente keinen metallischen Charekter haben.

Bei der zweiten Horizontalreihe besteht das innere Flektronensystem laut Tabelle auf Seite 192 aus 10 Elektronen, die sich möglichst symmetrisch um den Atomkern verteilen müssen, was geschieht, wenn sie in zwei Ringren von je 5 Elektronen um den Atornkern rotieren (Fig. 7). hommt bei den letzten vier Elementen dieser Reihe nun noch ein üußerer Elektronenring hinzu, so erfordert es die Symmetrie, dab dieser sich in der aus Fig. 7 a ersichtlichen Weise za den beiden Elektronenringen des inneren Elektronensystems einstellt. Der grade Kraftlinienweg vom Atomkern zu den Elektroneu

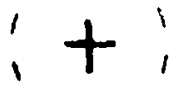

Fig. 7.

Schnitt durch die beiden inneren Elektronenringe der II. Horizontalreibe.

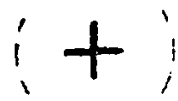

Fig. 8.

Schnitt durch die inneren 3 Elektronen. ringe der III. Horizontalreihe.

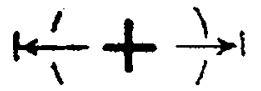

Fig. 7 a.

Stellung des änBeren Elektronenxinges in der II. Horizontalreibe.

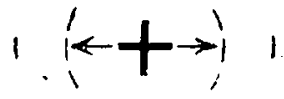

Fig. 8 a.

Stellung des änBeren Elektronenringes in der III. Borizontalreihe.

des äuB日ren Ringes geht dann zwischen den beiden inneren Elektronenringen hinduroh, so $\mathrm{da} B$ die Kraftlinien auch bei dieser Gruppierung der Flektronen ungestört zu den üußeren Elektronen gelangen und diese festhalten können. Daher haben dic rechtsstehenden Flemente auch in dieser Horizontalreiłse lieinen metsllis chen Charakter.

Bei der dritten Horizontalreihe besteht das innere Elektronensystem aus 18 Elektronen, die sich symmetrisch auf einer Kugelflächt verteilen können, wenn sie sich auf drei Ringe verteilen (Fig. 8), von denen der ruittlere 8 , die beiden äußeren je 5 Elektronen haben. Kommt dann noch ein äuBerer Elektronening hinzu, so mu $B$ dieser sich in der aus Fig. 8a ersichtlichen Weise zu den drei inneren Elektronenringen einzustellen suchen. Bei dieser Gruppierung bilden aber die 8 inneren Elektronen des mittleren inneren Ringes ein Hindernis für die Kraftlinien, die vom Atomkern zu den Elektronen des äu Buren Ringes gehen, so dab diese weniger stark vom Atomkern festgehalten werden und daher ausgestoben werden können. Aus diesem Grunde haben die betreffenden Elemente auf der rechten Seite der drit- 
ten Horizontalreihe einen mehr metallischen Charakter als die über ihnen stehenden Elomente in der ersten und zweiten Horizontalreihe.

Bei der. vierten Horizontalreihe besteht das innere Elektronensystem laut beigegebener Tabelle aus 28 Elektronen, die sich auf einer Kugelfläche symmetriech verteilen können, wenn sie sich derart auf 4 Ringe verteile $n$, da $B$ in den beiden inneren Ringen je 8 in den beiden äuBeren Ringen je 6 Elektronen kreisen. Der Symmetrie wegen müssen sich diese 4 Ringe in der ans Fig 9 ersichtlichen Weise zueinander und zum Atomkern einstellen. Kommt dann noch ein äuBerer Elektronenring hinzu, so muß dieser sich zu diesem System in der aus Fig. 9 a ersichtlichen Weise einstellen, bei welcher der direkte Kraftlinienweg wieder ungestört vom Atomkern zu den Elektronenkern des äuBeren Ringes verläuft. Infolgedessen werden die Elektronen des äuBeren Elektronenringes in der vierten

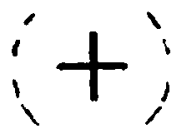

Fig. 9.

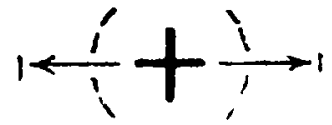

Fig. 9.

Schnitt durch die 4 inneren Elektronenringe der IV. Horizontalreihe.

Stellung des dußeren Elektronenringes in der IV. Horizontalreihe.

Horizontalreihe wieder fester vom Atomkern angezogen werden and die betreffenden Elemente am SchluB dieser Reihe haben wieder einen metalloiden Charakter.

Wenn das innere Elektronensystem bei der ersten Horizontalreihe aus einem Elektronenringe, bei der zweiten Horizontalreihe ans zwei Elektronenringen, bei der dritten Horizontalreibe aus drei Elektronenringen und bei der vierten Horizontalreihe aus vier Elektronenringen besteht, so darf inan wohl vermuten, da.B es bei der fünften Horizontalreihe aus 5 und bei der sechsten Horizontalreihe aus 6 Elektronenringen besteht.

Bei dem aus 5 Ringen bestehenden inneren Elektronensystem der fünften Horizontalreihe erfordert die Symmetrie, dab der äuBere Elektronenring sich in der aus Fig. 10 und 10a ersichtlichen Weise einstellt. Bei dieser Gıuppiezung wird ebenso wie bei der dritten Horizontalreihe der Kraftlinienweg zwischen dem Atomkern und dem än Beren Flektronenringe durch den mittleren Ring des inneren Elek. tronensystems gestört, was zur Folge hat, dab die rechtsstehenden Elemente bei der fünften Horizontalreihe ebenso wie bei der dritten einen metallischen Charakter haben. Dagegen kommt am rechten 
Ende der sechsten Horizontalreihe wiederun der metalloide Charakter zum Vorschein, weil sich dort der äußere Elektronenring zu den 6 inneren Elektronenringen in der aus Figg. 11 und 11 a ersichtlichen Weise derart einstellen muB, $\mathrm{da} B$ die Kraftlinien vom Atomkern zwischen den beiden mittleren Ringen des inneren Flektronensystems hindurch ungestört auf direktem Wege zu den Elcktronen des äuberen Ringes gelangen können. ${ }^{1}$ )

Durch die eben angestellten Betrachtungen dürfte nachgewlesen sein, daß die Elektronen des äußeren Elektronenringes in der ersten, zweiten, vierteu und sechsten Horizontalreihe vom Atomkern stärker

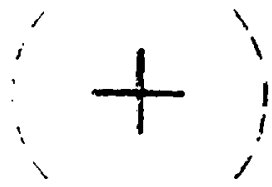

Fig. 10.

Schnitt durch die 5 inneren Elektronenringe der V. Horizontalreihe.
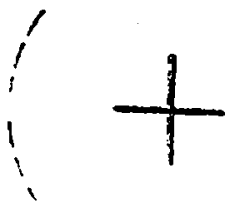

Fig. 11.

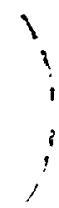

'

Schnitt durch die 6 inneren Elektronenringe der VI. Horizontalreihe.

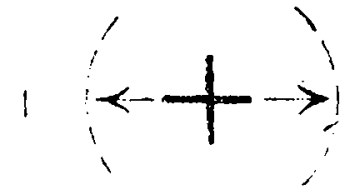

Fig. 102.

Stellung des suBeren Elektronenringes in der V. Horizontalreihe.

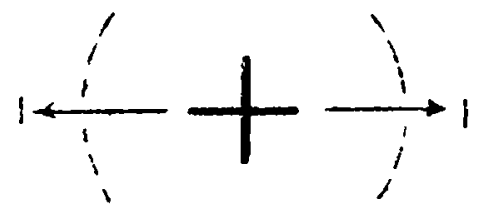

Fig. $11 \mathrm{a}$.

Stellung des ïuberen Elektronenringes in der VI. Horizontalreihe.

festgehalten werden als in der dritten und fünften Horizontalreihe. In diesen beiden Reihen sind daher die Elektronen des äußeren Elektronenringes relativ leicht herausstoßbar und verschiebbar, wodurch die rechtsstehenden Elemente in diesen beiden Horizontalreihen einen metallischen Charakter erhalten, während die ihnen entsprechenden Elemente der arsten, zweiten, vicrten und sechsten Horizontalreihe Metalloide sind.

Der metallische Charakter, der bei den rechtsstehenden Elementen der dritten und fünften Horizontalreilhe durch die Herausstoßbarkeit der Elektronen des üuBeren Elektronenringes hervor-

1) Der äußere Elektronenring nimmt die in den Figg. 83 und 10a gezeichnete Stellung allerdings nur bei isolierten Atomen ein; verbinden sich zwei Atome zu einom Molekïl, so verschiebt sich dieser auBere Eloktronenring in der weiter unten auf $\mathrm{S.207}$ beschriebenen und durch Figg. 13 und 14 veranschaulichten. Weise, wodurch aber die HerausstoBbarkeit der åußeren Elektronen nicht aufgehoben wird. 
gerufen wird, muB natürlich verschwinden, sobald die Elektronen des äuBeren Ringes von diesem fort in eine Valenzstellung gehen, was z. B. bei den höheren Oxyden des $\mathrm{Cr}$ und $\mathrm{Mn}$ der Fall ist. Deshalb zeigen diese beiden Metalle in ihren höheren Oxyden einen metalloiden Charakter, indem sie Säuren bilden. Die niederen Oxyde des Cr nnd Mn haben dagegen den basischen Charakter der Metalloxyde, weil bei ihnen Elektronen im äuBeren Elektronenringe des $\mathrm{Cr}$ und $\mathrm{Mn}$ vorhanden sind, die infolge ihrer wenig festen Bindung mit dem Atomkern den metallischen Charakter des $\mathrm{Cr}$ and $\mathrm{Mn}$ hervorrufen.

\section{Die Unterbrechnngen des periodischen Systems.}

Die Unterbrechungen des periodischen Systems bestehen darin, daB am Anfang der vierten, sechsten und zehnten Horizontalreihe der Tabelle auf S.192 das Edelgas fehlt, und daB jedesmal statt dessen eine Gruppe metallischer Elemente vorhanden ist, die nicht in die eigentlichen Perioden des Systems hineinpassen; es sind dies die Fe-, Ru- und Os-Gruppen.

Aus den Figg. 7 bis 11 läBt sich ableiten, weshalb am Anfang der vierten und sechsten Horizontalreihe keine Edelgase entstanden sind.

Die inneren Elektronensysteme der dritten und fünften Horizontalreihe (Figg. 8 und 10) unterscheiden sich von den inneren Elektronensystemen der vierten und sechsten Horizontalreihe (Figg. 9 und 11) dadurch, dab die beiden zuerst genannten Horizontalreihen einen mittleren Elektronenring haben, der am Äquator der Kugel liegt, während bei den inneren Elektronensystemen der vier ersten Elemente der vierten und sechsten Horizontalreihe keine Elektronen auf dem Äquator kreisen. Nun ist aber die Zentrifugalgeschwindigkeit bzw. die Zentrifugalkraft der am Äquator kreisenden Elektronen am größten. Da nun ferner die Beständigkeit des Elektronens ystems um so größer wird, je größer die Zentrifugalkraft der Elektronen ist, so müssen die Elektronensysteme der dritten und fünften Horizontalreihe infolge der größeren Zentrifugalgeschwindigkeit der an ihrem Äquator befindlichen Elektronen beständiger sein als die Elektronensysteme der vierten und sechsten Horizontalreihe. Bei den beiden zuletzt genannten Reihen sind daher die inneren Elehtronensysteme nicht so beständig, daB ihre Elektronen schon durch eine ihnen gleich große Anzahl positiver Ladungen am Atomkern in dem $\mathrm{Maße}$ festgehalten werden können, daß keine Elektronen vom Atomkern fort in eine Valenzstellung gehen können.

Aus diesem Grunde konnten sich die am Anfang der vierten und 
sechsten Horizontalreihe zu erwartenden Edelgase nicht bilden, und an ihrer Stelle haben sich andere Elemente gebildet, die nicht in die eigentlichen Perioden des Systems hineinpassen.

Das erste dieser Elemente ist das Eisen mit 26 negativen Elektronen und 26 positiven Ladungen am Atomkern. Von diesen 26 Elektronen können 6 Elektronen zu den Valenzelehtronen gehen, so daß für das innere Elelitronensystem des $\mathrm{Fe}$ nur 20 Elektronen übrig bleiben. Unter den eben beschriebenen Umständen, die am Anfang: der vierten Horizontalreihe auftreten, vermögen offenbar die 26 positiven Ladungen des Fe-Atomkerns nicht mehr als 20 Elektronen so fest zu einem inneren Elektronensystem zo verbinden, da $\$$ aus diesem keine Elektronen in die Valenzstellung gehen können. Das auf das Fe folgende $\mathrm{Ni}$ bildet mit seinen 27 positiven Ladungen und 27 Elektronen anscheinend ein inneres Elektronensystem von 23 Elektronen, während 4 Elektronen entweder in einen äußeren Elektronenring oder in eine Valenzstellung gehen. Das Co bildet anscheinend ein inneres Elektronensystem von 24 Elektronen, während wiederurn 4 Elektronen entweder in den äußeren Elelstronenring oder in eine Valenzstellung gehen.

$D_{a}$ das Kupfer auch zweiwertig auftritt, hat es zwei Valenz-lektronen, so da $B$ von seinen 29 Elektronen nur 27 fïr das innere Elektronensystem übrig bleiben. Das $\mathrm{Cu}$ gehört daher ebenfalls zu den Elementen der Eisengruppe und nicht in die vierte Horizontalreihe, deren Elemente laut Tabelle auf S. 192 ein inneres Elelstronensystem von 28 Elektronen haben. Bei der geringen Beständigkeit, the das Elektronensystem der vierten Horizontalreihe infolge Fehlens tines Ele litronenringes am Äquator zeigt, genügen offenbar die 29 positiven Ladungen, die der Atomkern des Cu besitzt, noch nicht, um alle 28 Elektronen in einem inneren System genügend stark festzuhalten. Dagegen paBt das $\mathrm{Zn}$ mit seinen zwei Valenzelektronen und 28 inneren Elektronen wieder in die regelmäBigen Perioden hinein. Demnach haben also die 30 positiven Ladungen am Atomkern des Zn genügend Kraft, um 28 innere Elektronen zu einem festen inneren System zu binden. Die auf das Zn folgenden Elemente zeigen ebenso wie dieses ein inneres Elektronensystem von 28 Elektronen und passen in die regelmäBigen Perioden des Systems binein.

Utber die vor Beginn der sechsten Horizontalreihe stehenden Elemente $\mathrm{Ru}, \mathrm{Rh}, \mathrm{Pd}$ lassen sich ganz gleichartige Betrachtungen anstellen und gleichartige Sehlüsse ziehen, wie eben über die Elemente $\mathrm{Fe}, \mathrm{Ni}$, Co angestellt wurder. 
Wenn wir über die selteneren, zum Teil wenig bekannten Ellemente, die in die achte und neunte Horizontalreihe gehören, hinwegsehen, so finden wir vor Anfang der zehnten Horizontalreihe noch eine weitere Unterbrechung, in der die Elemente Os, Ir, Pt stehen. Auch diese Unterbrechung ist auf die gleiche innere Ursache zuräckzuführen, wie die Unterbrechungen vor der vierten und sechsten Horizontalreihe. Denn auch bei der zehnten Horizontalreihe sind am Äquator der das Atom umgebenden Kugelfläche keine Elektronen vorhanden, deren große Zentrifugalgeschwindigkeit das System beständig macht. Vielmiehr müssen der Symmetrie wegen 5 Elektronenringe auf der einen Seite des Äquators und 5 Elektronenringe auf der anderen Seite des Aquators liegen, so daßjer Bildung des inneren Elektronensystems bei der zehnten Horizontalreihe die gleichen Schwierigkeiten entgegenstehen, wie bei der vierten und sechsten Horizontalreihe.

Da das Au dreiwertig auftritt und somit 3 Valenzelektronen besitzt, gehört es nicht an die Stelle der zehnten Horizontalreihe, an die man es bisher zu. stellen pflegte, sondern zu den Metallen Os, Ir, Pt. In Analogie mit dem Au. und dem $\mathrm{Cu}$ ist dann auch noch das $\mathrm{Ag}$ zu den Elementen $\mathrm{Ru}, \mathrm{Rh}, \mathrm{Pd}$ zu stellen.

\section{Die sich aus der Blektronentheorie ergebenden Klassen der chemisohen Elemente.}

Die im Vorhergehenden entwickelte Theorie ging davon aus, daß die Atome der chemischen Elemente aus Elektronen anfgebaut sind; dabei worde unterschieden zwischen Valenzelektronen, die vom Atomkern fort in eine Valenzstellung gehen können, und zwischen einem inneren Elektronensystem, dessen Elektronen so fest mit dem Atomkern verbunden sind, daß sie von demselben nicht fortgehen und in eine Valenzstellung treten können. Infolge dieser beiden verschiedenen Arten von Elektronen wird die Bildung von drei verschiedenen Klassen chemischer Elemente möglich, nämlich:

1. Elemente, welche kein inneres Elektronensystem, sondern nur. Valenzelektronen haben. Zu dieser Klasse gehört nur ein einziges Element, nämlich der Wasserstoff.

2. Elemente, welche nur ein inneres Elektronensystem und keine Valenzelektronen haben. Zu dieser Klasse gehören die Edelgase, die nur einatomig auftreten und keine Verbindungen eingehen können, weil sie eben keine Valenzelektronen haben.

3. Elemente, welche sowohl ein inneres Elektronensystem als auch Valenzelektronen haben. $7 /$ dieser Klasse gehören die Metalle und Metalloide. 
Bei den Metalloiden sind die Elektronen des inneren Elektronensystems so fest mit dem Atomkern verbunden, daB naoh dem Fortgang der Valenzelektronen keine Elektronen mehr aus dem Atom herausgestoßen werden können. Da* gegen entstehen die Metalle dadurch, dab Elektronen durch irgendwelche Ursachen aus den Atom herausstoBbar gemacht werden. Die hierbei in Betracht kommenden Ursachen sind im Vorhergehenden wie folgt abgeleitet:

a) Die linksstehenden Metalle (Alkalimetalle, alkalische Erden nsw.) erhalten ihren metallischen Charaliter dadurch, dab bei ihnen Elektronen des inneren Elektronensystems infolge ihrer geringen Zentrifugalkraft am Atomkern verschoben und aus dem Atom herausgestoßen werden können, wie im Kapitel 3 abgeleitet.

b) Die rechtsstehenden Metalle der dritten und fünften Horizontalreihe (V, $\mathrm{Cr}, \mathrm{Mn}, \mathrm{Mo}$ ) erhalten ihren metallischen Charakterdadurch, dab bei ihnen Elektronen des äuBeren Elektronenringes am Atomkern verschoben und aus dem Atom heransgesto Ben werden können, wie ebenfalls im Kapitel 3 abgeleitet.

c) Bei den auBerhalb der eigentlichen Perioden stehenden Me• tallen

$\begin{array}{llll}\mathrm{Fe}, & \mathrm{Ni}, & \mathrm{Co}, & \mathrm{Cu}, \\ \mathrm{Ru}, & \mathrm{Rh}, & \mathrm{Pd}, & \mathrm{Ag}, \\ \mathrm{Os}, & \mathrm{I}, & \mathrm{Pt}, & \mathrm{Au} .\end{array}$

ist nach den Ausführungen des Kapitels 4 das innere Elektronensystem relativ wenig beständig und außerdem ist auch noch ein äußerer Elektronenring vorhanden. Daher können Elektronen sowohl aus den inneren Elektronensystem als auch aus dem äußeren Elektronenring gestoßen werden, weshalb der metallische Charakter bei diesen Elementen besonders stark entwickelt ist.

d) Auch bei den in der vierten und sechsten Horizontalreihe linksstehenden Elementen (Zn und Cd) ist der metallisehe Charakter stark entwickelt, weil keiner der Elektronenringe ihres inneren Elektronensystems auf dem Äquator der das Atom umgebenden Elektronenkugelfläche liegt. Diese Ursache, durch welche die Elektronen nach den Ausführungen des Kapitels 4 eine geringere Durchschnittszentrifugalkraft erhalten und daher leichter aus dem Atom herausgestoBen werden können, fällt bei den in diesen beiden Reihen rechts stehenden Elementen fort, weil dort der äußere Elektronenring binzukommt, so dab wieder eine ungerade Zahl Elektronenringe vorhanden ist, von denen sich der mittlere am Äquator einstellen mub. 
e) Je größer die Zahl der zu einem Atom gehörenden Elektronen wird, je größer wird auch die Wahrscheinlichkeit, daß eine mehr oder minder große Anzahl Elektronen aus dem Atom herausgestoßen werden kann. Deshalb nimmt der metallische Charakter der Elemente mit wachsender Elektronenzahl bzw. mit wachsendem Atomgewicht in den verschiedenen Vertikalreihen regelmäBig zu und ron der siebenten Horizontalreihe ab haben alle Elemente (mit Ausnahme der Edelgase) einen metallischen Charakter.

Bei den Elementen, die rechts in den Perioden stehen, haben die Elektronen (wie im Kapitel 3 abgeleitet) eine große Zentrifugalkraft und sind daher nicht leicht herausstoßbar. Diese Elemente haben daher einen metalloiden Charakter, wenn nicht Elektronen durch sekundäre Einflüsse heraussto Bbar gemacht werden, was bei den eben unter b) und e) genannten Elementen der Fall ist.

Die in der ersten Horizontalreihe stehenden Elemente unterscheiden sich von den anderen Elementen dadurch, daB sämtliche den Atomkern umkreisenden Elektronen mit diesem in einer Ebene liegen, so $\mathrm{da} B$ der Atomkern nicht von einer aus Elektronen gebildeten Kugelfläche umgeben wird. Dies hat zur Folge, daB ein größerer Teil der von dem Atomkern ausgehenden Kraftlinien zu den auBerhalb des eigentlichen Atoms befindlichen Valenzelektronen gelangen kann als bei den Elementen der folgenden Horizontalreihen, bei denen der Atomkern von mehreren auf einer Kugelfläche liegenden Elektronenringen umgeben ist, vgl. Fig. 1. Infolgedessen ist die Fähigkeit, chemische Verbindungen mit anderen Elementen einzugehen, bei den Elementen der ersten Horizontalreihe am größten. Je größer die Zahl der den Atomkern umkreisenden Elektronenringe in den folgenden Horizontalreihen wird, desto weniger Kraftlinien können vom Atomkern zu den außerhalb des Atoms kreisenden Valenzelektronen gelangen und infolgedessen verringert sich die Tendenz chemische Verbindungen einzugehen in der Regel von Horizontalreihe zu Horizontalreihe.

\section{Atomvolumina.}

Die Größe der Atomvolumina wird in erster Linie dadurch geregelt, dab die Zahl der positiven Ladungen am Atomkern innerhalb jeder Horizontalreihe von links nach rechts zunimmt, während die Zahl der Elektronen des inneren Elektronensystems innerhalb jeder Horizontalreihe konstant bleibt. Infolge dieser Zunahme der positiven Ladungen müssen die Elektronen des inneren Elektronen- 
systems am so näher an den Atomkern herangezogen werden, je mehr das betreffende Element rechts steht. Der Umfang eines Atoms müBte daher bei den Elementen einer Horizontalreihe regelmäBig von links nach rechts abnehmen, wenn nicht noch andere Umstánde hinzukämen. Von besonderer Bederitung ist dabei der bei den rechts stehenden Elementen jeder Horizontalreihe nooh hinzutretende änBere Elektronenring. Dieser vermehrt natürlich den Umfang der Atome, wie ein Vergleich der Fig. 4 mit Fig. 5, der Fig. 7 mit $7 \mathrm{a}$, der Fig. 8 mit $8 \mathrm{a}$ us w. ohne weiteres erkennen läBt. Die Vermehrung

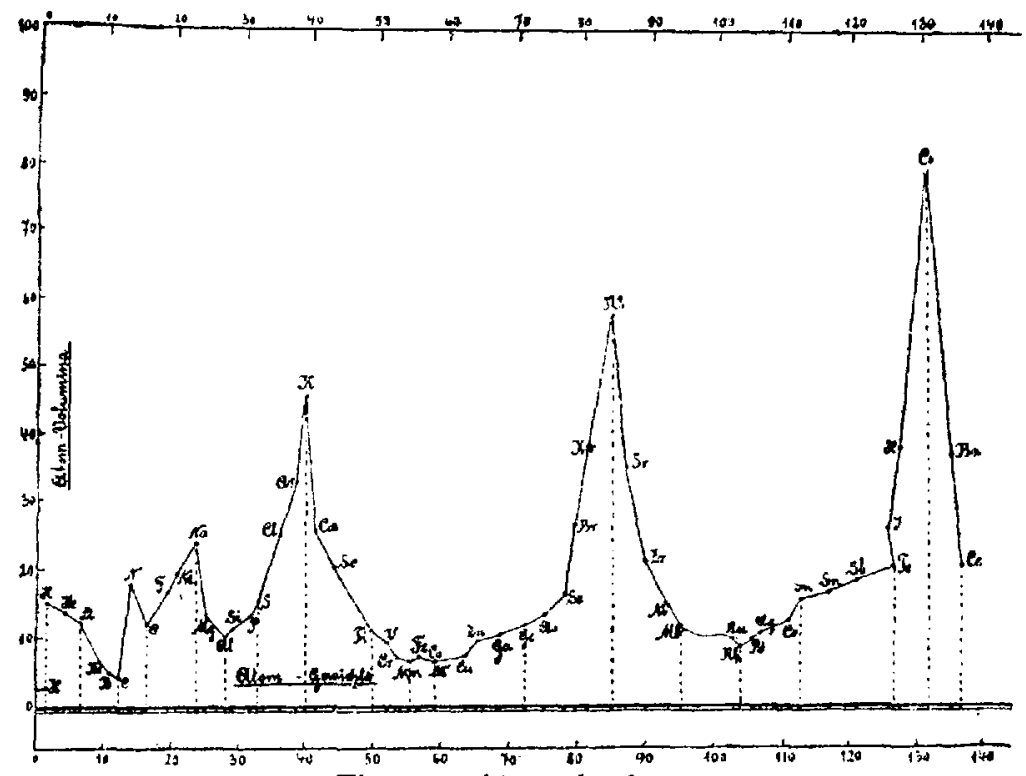

Fig. 12. Atomvolumina.

des Atomvolumens durch den äußeren Elektronenring wird um so größer, je mehr Elektronen er enthält. Bei zunehmender Zahl der an Atom. zurückbleibenden Valenzelektronen können diese sich event. auch in mehreren konzentrischen Ringen einstellen. die aber alle in der Mittelebene des Atoms liegen müssen. Wie die Kurve in Fig. 12 zeigt, tritt diese Zunahme des Atomvolumens deutlich in Erseheinung bei den letzten Gliedern der ersten Horizontalreihe $(\mathrm{N}, \mathrm{O}, \mathrm{F})$, der zweiten Horizontalreihe ( $\mathrm{Si}, \mathrm{P}, \mathrm{S}, \mathrm{Cl}$ ), der vierten Horizontalreihe (Ge, $\mathrm{As}, \mathrm{Se}, \mathrm{Br}$ ) und der sechsten Horizontalreihe ( $\mathrm{Sn}, \mathrm{Sb}, \mathrm{Te}, J$ ). Dagegen fehlt die zu erwartende Zunahme des Atomvolumens bei der dritten und fünften Horizontalreihe. Auch dieser Unterscbied zwischen der zweiten, vierten und sechsten Horizontalreihe einerseits und der dritten und 
fünften Horizontalreihe andererseits ist darauf zurückzuführen, daB dieser äuBere Elektronenring nur bei den Elementen der dritten und fünften Horizontalreihe leicht verschoben werden kann.

Verbinden sich 2 Atome dieser Elemente zu einem Molekül, so wird der leicht verschiebbare äuBere Elektronenring durch die $\mathbf{A b}$ stoBungskräfte, die die Elektronen dieser beiden Atome ausüben, bis an das äuBerste Ende seines Atoms geschoben (Figg. 13 und 14), wo er in das Bereich der vom Atomkern ausgehenden positiven Kraftlinien gelangt, von diesen festgehalten und durch engere Zusammenziehung seiner Elektronen an seinem Umfang vermindert wird. ${ }^{\text {) }}$ ) Aus diesem Grunde trägt der äußere Elektronenring bei den Elementen der dritten und fünften Horizontalreihe, bei denen er in der

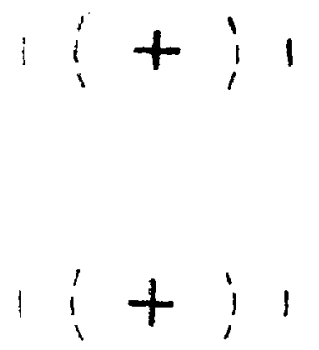

Fig. 13.

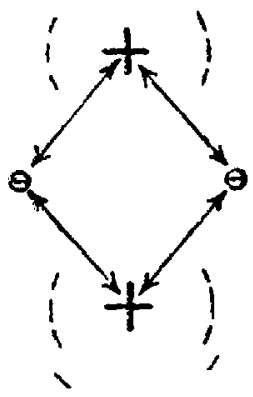

Fig. 14.

Verschiebung des äuBeren Elektronenringes der III. Horizontalreihe aus der Stellung, die er bei Einzelatomen einnimmt (Fig. 13), in die Stellang, die er bei zu Molekülen verbundenen Atomen einnimmt (Fig. 14).

eben beschriebenen Weise versehoben werden kann, nicht viel zur Vermehrung des Atomvolumens bei. In diesen beiden Horizontalreihen kommt daher das oben abgeleitete Grundgesetz, nach dem das Atomvolumen bei den Elementen einer Horizontalreibe von links nach rechts stündig abnehmen muß, dextlich zum Ausdruck.

$\mathrm{DaB}$ die in den linken Hälften der IV. und VI. Horizontalreihe stehenden Elemente so geringe Atomvolumina haben, ist wohl węnigstens zum Teil zurückzuführen auf das Fehlen eines am Äquator stehenden Elektronenringes. Die geringen Atomvolumina der aulerhalb der eigentlichen Perioden stehenden Elemente der Fe-, Ru - und Os-Gruppe deuten ebenfalls darauf hin, daB auch bei diesen Ele-

1) Diese Verminderung des Ringdurchmessers ist jedoch nicht so stark, daß dadurch die HerausstoBbarkeit seiner Elektronen aufgehoben wïrde, durch welche der metallische Charakter bei cliesen Elementen gemä $B$ den Ausfübrungen im Kapitel 3 hervorgerufen wird. 
menten kein Elektronenring an Äquator vorhanden ist. Außerdem spricht wohl noch folgender Umstan 1 mit:

Bei den genannten metallischen Elementen hat die leichte Verschiebbarkeit der Elektronen zur Folge, daß sich leicht einige Elelitronen eines Moleküls einigen positiven Stellen eines benachbarten Moleküls gegenüberstellen können, wodurch die Moleküle stärker aneinander gezogen und die $\mathrm{Zahl}$ der in der Volumeinheit enthaltenen Moleküle vergrößert wird, so daß die Größe des Volumens, das auf das einzelne Molekül bzw. auf die in ihm enthaltenen Atome entfällt, vermindert wird. Ebenso sind auch bei der vierten und sechsten Horizontalreihe die Elektronen des inneren Elektronensystems wegen ihrer geringen Durchschnittsfugalkraft (Kapitel 4) leicht verschiebbar, weshalb auch die in den linken Hälften dieser Reihen stehenden Elemente ( $\mathrm{Zn}$ und $\mathrm{Ga}_{\mathrm{a}}$ sowie $\mathrm{Cd}$ und $\mathrm{J}_{\mathrm{n}}$ ) kleine Atomvolumina zeigen, während in den rechten Hälften dieser Reihen die Atomvolumina durch den in diesen Reihen nicht verschiebbaren ïu $\beta_{t}$ ren Elektronenring vergrößert werden.

Da die Edelgase am meisten links stehen, müBten sie eigentlich die größten Atomvolumina aufweisen. Tatsächlich aber werden die Atomvolumina der Edelgase dadurch vermindert, da $\beta$ bei diesen die Valenzelektronen fehlen. Letztere vermehren die Atomvolumina um so mehr, je größer die Entfernung ist, in der sie sich von den Atomkernen einstellen. Diese Fntfernung ist bei den Elementen der ersten Horizontalreihe am kleinsten, weil das innere Elektronensystem bei dieser nur zwei Elektronen hat, die infolge ihrer geringen Anzanl auch nur geringe AbstoBungskräfte auf die Valenzelektronen ausüben. Dagegen wächst die Stärke dieser Abstoßung und damit die Entfernung, in der die Valenzelektronen sich von den Atomlsernen einstellen, mit zunehmender Elektronenzahl von Horizontalreihe zu Horizontalreihe. In der ersten Horizontalreihe wird daher das Atomvolumen des $\mathrm{Li}$ nur so wenig vermehrt, dab es immerhin noch kleiner bleibt als das des He. In den folgenden Horizontalreihen steigt aber die durch die Valenzelektronen bewirkte Zunahme der Atomvolumina derart, daß die Atomvolumina von $\mathrm{K}, \mathrm{Rb}$ und $\mathrm{Cs}$ erheblich größer als die der vorhergehenden Edelgase werden.

Charlottenburg. IV.

Bei der Redaktion eingegangen am 22. Januar 1919. 